\title{
Japan Advanced Materials Science and Engineering Society Holds Inaugural Symposium
}

A ceremonial symposium on March 16-17, 1989 at the Keio Plaza Hotel in Tokyo inaugurated Japan's Advanced Materials Science and Engineering Society (AMSES). The new society emphasizes an interdisciplinary approach to materials science and engineering.

Prof. M. Doyama of Nagoya University is president of the new Society, and Profs. S. Somiya of the Nishi Tokyo University and M. Hasegawa of the University of Tokyo are vice presidents.

The Society plans to hold two annual meetings per year and to produce a regular newsletter for members who already number more than 150. A corporate affiliates program has also been established and presently consists of more than two dozen members.

\section{Interdisciplinary Materials Research Needed}

The event that sparked the eventual formation of AMSES was the first International MRS Conference on Advanced Materials, which attracted about 1,600 attendees to Tokyo in June 1988. That meet-

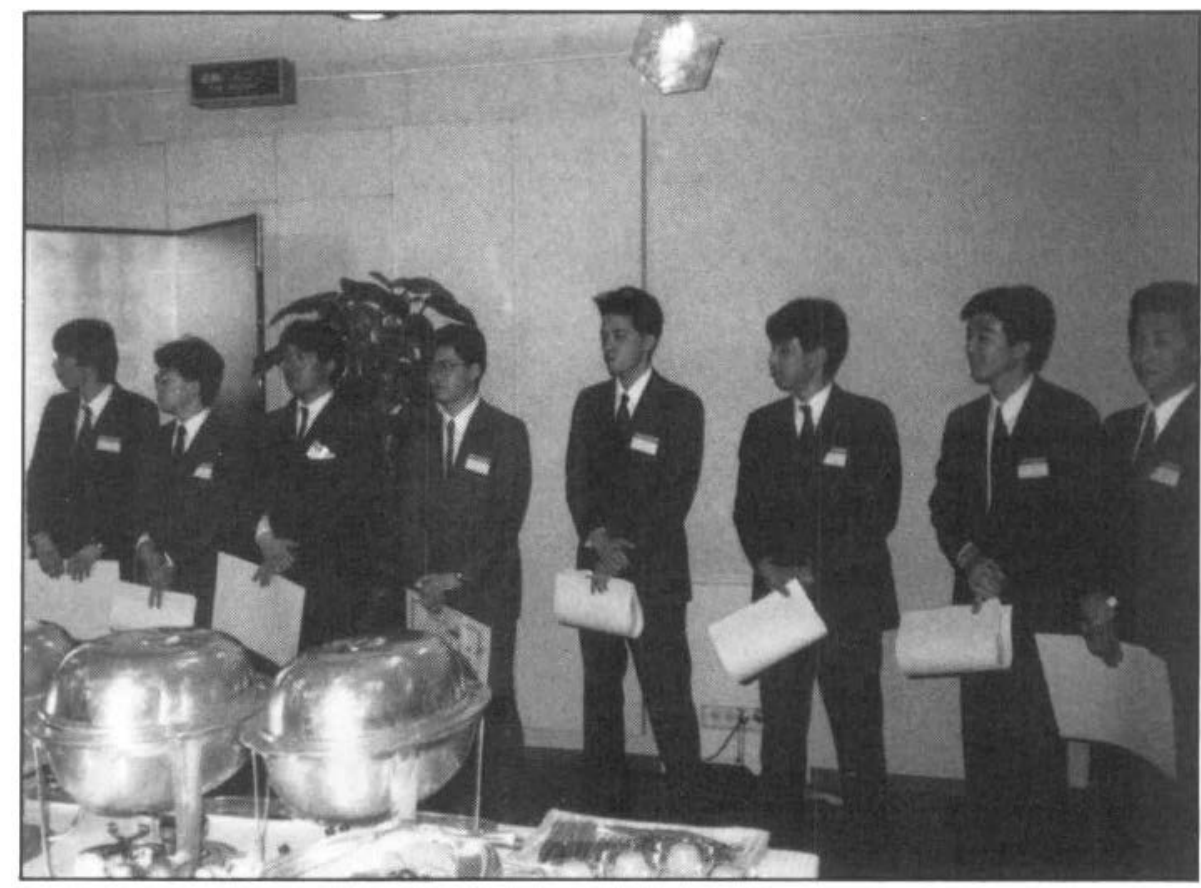

Student award winners are recognized at the AMSES inaugural symposium.

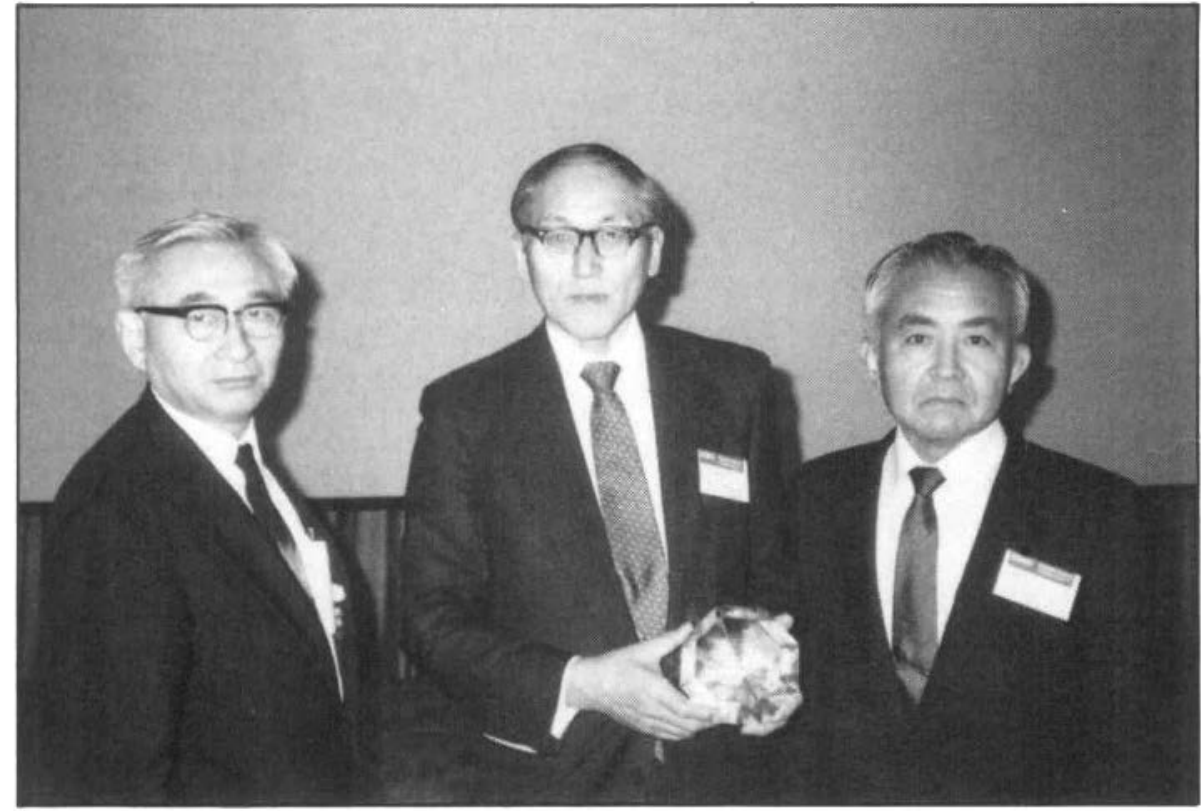

AMSES President M. Doyama (center) displays diamond plaque, accompanied by Vice President $S$. Sōmiya (left) and $M$. Hasegawa. ing was organized primarily by scientists and engineers in Japan under the leadership of Profs. Sōmiya and Doyama. The success of the 16-symposia meeting led to a recognition of the need for an interdisciplinary materials research society in Japan.

The vast majority of the hundreds of scientific societies in Japan are all single discipline in character. AMSES represents a significant departure from this trend.

The Society's founders also surmounted major obstacles associated both with the complexity of registration with the Japanese Ministry of Education, Science and Culture (Monbusho) and with the requirement to have cash reserves (about $\$ 320,000)$ in the bank in less than nine months. Scientific research in Japanese universities is under the control of Monbusho and an important degree of prestige accrues to organizations registered with it.

\section{Inaugural Symposium}

The March inaugural ceremony featured three parallel sessions (two oral and one poster). Of particular note were presenta- 


\section{President Doyama's Address to the Advanced Materials Science and Engineering Society of Japan}

As advanced technology develops toward its highest goals, a small improvement in existing materials is not enough to meet the demands. The deadlock of advanced technology often brings the invention of new materials.

Human civilization has grown along with materials. The Stone Age, the Bronze Age, and the Iron Age represent the materials most used in those times. Since the beginning of the 20th century, the plastic age, the semiconductor age, the new ceramics age, and the composite materials age have been identified, but no single material dominates.

In addition to the traditional classification of materials (the warp) such as metals, semiconductors, ceramics and organic materials, materials have to be studied by the woof. After World War II, metallurgy hit a deadlock. To overcome the deadlock, metallurgy changed to materials science, absorbing the knowledge of physics, chemistry, chemical engineering, electrical engineering, mechanical engineering, civil engineering, etc., and collaborating with ceramics, semiconductors, and organic materials.

This movement was not successful in Japan because at that time the production of iron and steel in Japan was increasing very rapidly, and the country could not spare a sidelong glance at other fields. Now the iron and steel industry in Japan has reached maturity, and the value of materials science is being rediscovered in Japan. In organic materials, the properties of single molecules reflect those of the entire product. Synthesis is emphasized. Fracture can be treated by itself or in comparison among metals, semiconductors, ceramics and organic, rheology and amorphous materials. The complexity of the problem cannot be adequately addressed by traditional societies.

Process, properties, structure, and environment are the four elements of materials. Without good processes, good materials cannot be made. In the future, the development of materials must be made from a broad perspective and must be useful for mankind.
The Materials Research Society, begun in the United States, has grown rapidly with the strategy of materials science and flexibility. The MRS International Conference on Advanced Materials held in Tokyo, Japan drew 1,600 participants and proved a great success. The Advanced Materials and Engineering Society of Japan was established by the kind invitation of MRS President R.P.H. Chang through the collaboration of Prof. Somiya and Dr. K. Inoeu. This Society is an international society holding a strong connection with MRS.

On the occasion of the founding of the Advanced Materials Science and Engineering Society, we respectfully request your guidance and warm support.

Masao Doyama

President

Advanced Materials Science

and Engineering Society

Professor, Nagoya University,

Emeritus Professor, University of Tokyo tions by Prof. Emeritus R.R. Hasiguti of the University of Tokyo on "Fusion Reactor Materials" and by Dr. H.G. Rammrath on

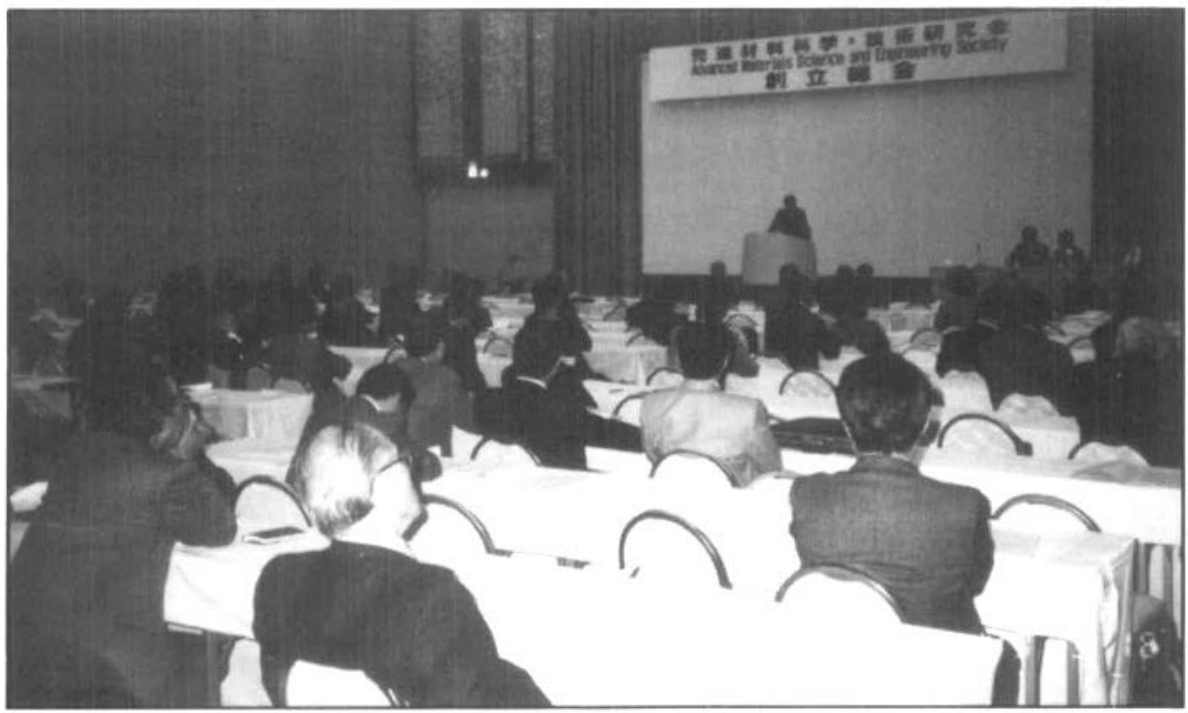

Inaugural symposium of the Advanced Materials Science and Engineering Society.
"The Future of Engineering Plastics: One View," which were part of a special ceremonial session.
Poster sessions, including "best poster" competitions, displayed 12 from undergraduate students (with 4 awards); 21 from master's degree candidates (with 7 awards); 7 from doctoral candidates (with 3 awards); 6 from researchers under 35 years of age (with 2 awards); and 13 other posters. The oral sessions included both invited and contributed papers. A total of 220 individuals, including participants from six countries, as well as 35 industrial representatives attended the meeting.

One of the international delegates, 1989 MRS President R.P.H. Chang, commented: "It was a great experience for me to attend the inaugural ceremony meeting. I was particularly impressed that the organizers placed strong emphasis on student participation. In fact, they told me future meeting schedules would be organized so that most students could participate." Chang noted that student participation "is a matter of great significance which other societies might emulate because for a field to grow, we need to train future leaders."

On behalf of MRS, Chang extended best wishes for success to AMSES. He also presented President Doyama with a diamondshaped commemorative plaque. 